\title{
Determining Reflectance and Light Position from a Single Image Without Distant Illumination Assumption
}

\author{
Kenji Hara ${ }^{\dagger, \S}$ \\ $\dagger$ Fukuoka Industrial \\ Technology Center \\ hara@fitc.pref.fukuoka.jp
}

\author{
Ko Nishino ${ }^{\ddagger}$ \\ $\ddagger$ Department of Computer Science \\ Columbia University \\ kon@cs.columbia.edu
}

\author{
Katsushi Ikeuchi ${ }^{\S}$ \\ $\S$ Department of Computer Science \\ The University of Tokyo \\ ki@cvl.iis.u-tokyo.ac.jp
}

\begin{abstract}
Several techniques have been developed for recovering reflectance properties of real surfaces under unknown illumination conditions. However, in most cases, those techniques assume that the light sources are located at inifinity, which cannot be applied to, for example, photometric modeling of indoor environments. In this paper, we propose two methods to estimate the surface reflectance property of an object, as well as the position of a light source from a single image without the distant illumination assumption. Given a color image of an object with specular reflection as an input, the first method estimates the light source position by fitting to the Lambertian diffuse component, while separating the specular and diffuse components by using an iterative relaxation scheme. Moreover, we extend the above method by using a single specular image as an input, thus removing its constraints on the diffuse reflectance property and the number of light sources. This method simultaneously recovers the reflectance properties and the light source positions by optimizing the linearity of a log-transformed Torrance-Sparrow model. By estimating the object's reflectance property and the light source position, we can freely generate synthetic images of the target object under arbitrary source directions and source-surface distances.
\end{abstract}

\section{Introduction}

Several methods have been developed to simultaneously recover image surface reflectance properties and the lighting. These techniques can be divided into two categories: techniques using a set of images $[2,3,4]$ and a single image $[1,5,6]$ as inputs with the exception of $3 \mathrm{D}$ geometric models of the object. In the latter category, for example, Ikeuchi et al. [1] developed an algorithm to determine both the surface reflectance properties and the light source di-

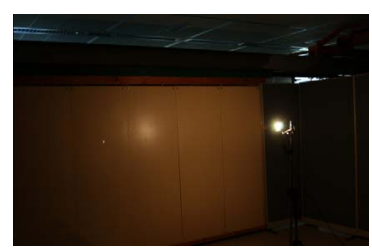

(a)

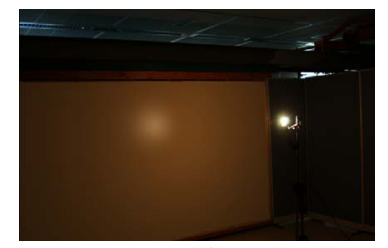

(b)
Figure 1. An example of photometric modeling of indoor environment: (a)real image; (b)synthesized image.

rection from a single image based on analysis of a simplified Torrance-Sparrow reflection model (called T-S model) [12]. I. Sato et al. [6] proposed a method for estimating the complex illumination distribution of a real scene by using a radiance distribution of inside shadows cast by a real object. Tominaga et al. [5] adopted the Phong model to determine the surface reflectance properties and the direction of illumination from a single color image. However, all of these methods assume that the light and viewing positions are distant, and thus follow parallel illumination and orthogonal projection; therefore, they do not offer a solution for real scenes under light and viewing positions at finite distances. In general, the distant illumination assumption is not correct if the light source-surface distance is not very large compared with that of the surface area, e.g., reflectance modeling of indoor environments (Fig.1). One of the drawbacks of using the distant illumination assumption is when the input is a single image of a planar surface. In this case surface normals, light directions and viewing directions at all surface points produce the same angle. As a result, no fitting process can be applied.

Recently, a method similar to the method proposed in this paper has been introduced to recover the reflectance properties of multiple objects from a single image [7]. While we take a similar approach to separate the image into regions according to their type of reflection and fit reflection models to each regions, our method also recovers the 
light source position and the reflectance properties of textured surfaces with specular reflection.

Our goal is to recover the light source position and surface reflectance properties from a single object image captured under a single light source at a finite distance; and then to create a new photo-realistic synthetic image under novel light source conditions with not only source direction modification but also light source-surface distance modification. Given a single color image that has specular regions, our first method consists of two main aspects. First, we determine rough diffuse regions, and the peak pixels of the whole image, which is usually located at the specular region. From these peak pixels and rough diffuse region, we initialize the values of the light source direction and source-surface distance, respectively, as well as Lambertian diffuse parameters. Then, based on these initial values, we simultaneously estimate the actual values of the light source position and reflectance properties using an iterative separating-andfitting relaxation technique. However, this recovery method is largely limited to the following: (1) the diffuse reflection property is homogeneous Lambertian, (2) the specular peak must be visible somewhere on the surface of the target object while avoiding saturation.

We attempt to extend the above method by removing these restrictions, and presenting an improved method for recovering the reflectance property and the light source position, assuming only that the target object has a homogeneous specular reflection property. First, polarization filters are used for separating the specular and diffuse reflection components [13]. Given the obtained single specular image and 3D geometric model of an object as an input, the second method initially estimates the light source position and the specular reflection parameters based on a linearized $\mathrm{T}-\mathrm{S}$ reflection model. These parameters are then refined by using the original T-S reflection model.

The main advantages of our methods are: (1) the inputs are only a single image and a 3D geometric model of the scene, (2) unlike past methods, we estimate the three dimensional light source positions as well. In particular, even under multiple light sources, our second method allows the recovery of the position of each light source and the reflectance properties of textured surfaces.

\section{Reflection Model}

In this section, we give a brief overview of the reflection model used in our method. Generally, reflection models are described by linear combinations of two reflection components: the diffuse and the specular reflections. This model was formally introduced by Shafer [8] as the dichromatic reflection model. The diffuse reflection component represents reflected rays arising from internal scattering inside the surface medium. The specular reflection component, on the other hand, represents light rays immediately reflected on the object surface.

Specifically, we use the T-S reflection model [12] by assuming that the Fresnel reflectance coefficient is constant and the geometric attenuation factor is 1 . Using this reflection model, the specular reflection of an object's surface point is given as

$$
I_{c}=\left[k_{d, c} \cos \theta_{i}+\frac{k_{s, c}}{\cos \theta_{r}} \exp \left[-\frac{\alpha^{2}}{2 \sigma^{2}}\right]\right] L_{c}, c=R, G, B
$$

where index $c$ represents $\mathrm{R}, \mathrm{G}$ and B component, $I_{c}$ is the image pixel value, $\theta_{i}$ is the angle between the light source direction and the surface normal, $\theta_{r}$ is the angle between the viewing direction and the surface normal, $\alpha$ is the angle between the surface normal and the bisector of the viewing direction and the light source direction. $L_{c}$ is given by:

$$
L_{c}=\frac{L_{q, c}}{r^{2}}, \quad c=R, G, B
$$

where $L_{q, c}$ is the radiance of the point light source in each color band and $r$ is the distance between the point light source and the object surface point.

$k_{d, c}$ and $k_{s, c}$ are coefficients for the diffuse and specular reflection components respectively, and $\sigma$ is the surface roughness measured as the standard deviation of microfacet orientations. Using a 3D geometric model and by accomplishing camera calibration, the angle $\theta_{r}$ can be obtained for each image pixel. Angles $\alpha$ and $\theta_{i}$ are unknown, since these angles require the light source direction, which is unknown at this moment.

In (1) we can observe that the values of $\left(k_{d, c}, k_{s, c}\right)$ and $L_{c}$ cannot be separated by only knowing the value of $I_{c}$. Thus, we redefine the reflection parameters as:

$$
K_{d, c}=k_{d, c} L_{q, c}, \quad K_{s, c}=k_{s, c} L_{q, c}, \quad c=R, G, B
$$

With these definitions, we can rewrite (1) as

$$
I_{c}=\frac{K_{d, c} \cos \theta_{i}}{r^{2}}+\frac{K_{s, c}}{r^{2} \cos \theta_{r}} \exp \left[-\frac{\alpha^{2}}{2 \sigma^{2}}\right], c=R, G, B
$$

In this paper, we refer to $\tilde{K}_{s}=\left(K_{s, R}, K_{s, G}, K_{s, B}\right)$ and $\sigma$ as the specular reflection parameters, and $\tilde{K}_{d}=$ $\left(K_{d, R}, K_{d, G}, K_{d, B}\right)$ as the diffuse reflection parameters. Note that $\tilde{K}_{d}$ and $\tilde{K}_{s}$ contain the light source intensity $\tilde{L}_{q}=\left(L_{q, R}, L_{q, G}, L_{q, B}\right)$. In Section 3 and 4 , we remove the subscript $c$, as the same computation is executed independently for each color band. If the estimated roughness parameters do not have the same value from the three color channels, we determine the final roughness parameter by calculating an average of those values.

\section{Inverse Rendering from a Single Color Im- age}

We now describe the algorithm for estimating the position of the light source, and the reflectance parameters from 
a single color image and a 3D geometric model of the object. In this method, we assume that: (1) the camera parameter is known, (2) interreflection, shadows, and saturated pixel values are avoided, (3) the scene is illuminated by a single point light source, (4) at least one specular peak is visible on the surface of the target object, (5) the surface reflection can be modelled as a linear combination of the Lambertian diffuse model and the T-S specular model.

\subsection{Light Source Distance}

The light source direction at the object's surface point $P$ satisfying $\alpha=0$ can be written as

$$
\boldsymbol{L}_{p}=\boldsymbol{N}_{p}+\left(\boldsymbol{N}_{p}, \boldsymbol{V}_{p}\right) \boldsymbol{N}_{p}-\boldsymbol{V}_{p}
$$

where $\boldsymbol{L}_{p}$ is a unit vector with a light source direction and a starting point $P, \boldsymbol{V}_{p}$ is a unit vector with a viewing direction and a starting point $P$, and $\boldsymbol{N}_{p}$ is a unit normal vector at $P$. Then, the location vector $\boldsymbol{L}$ of the light source can be expressed as

$$
\boldsymbol{L}=\boldsymbol{P}+t \boldsymbol{L}_{p}
$$

where $\boldsymbol{P}$ is the location vector of $P$ and $t$ is the distance between $\boldsymbol{P}$ and $\boldsymbol{L}$.

We can regard the specular peak $\left(i_{p}, j_{p}\right)$ as the pixel location of $P^{1}$. As a consequence, once we obtain $\left(i_{p}, j_{p}\right)$, $\boldsymbol{P}$ can be calculated using the camera projection matrix, which makes $\boldsymbol{L}_{p}$ directly computable from (5). However, to estimate the light source position, we also need the value of $t$ (the light source distance), which is unknown. In the next subsection, we describe how to estimate $t$ and the reflectance parameters in (4) simultaneously.

\subsection{Model Fitting to Lambertian Component}

To obtain the initial estimates of the light source distance and reflectance parameters, we need to perform the following two steps: First, we extract the diffuse region $\Omega_{d}$

$$
\Omega_{d}=\left\{(i, j) \in \Omega \mid\left(i-i_{p}\right)^{2}+\left(j-j_{p}\right)^{2}>T^{2}\right\}
$$

where $\Omega$ is the set of $2 \mathrm{D}$ points of object's surface points and $T$ is a positive integer concerning the radius of the specular region. Then, we determine the light source distance $t$, by fitting the diffuse term of (4) to the diffuse region, which means

$$
t^{*}=\underset{t}{\operatorname{argmin}} \sum_{(i, j) \in \Omega_{d}}\left[u(i, j, t)-\frac{1}{N_{d}} \sum_{(i, j) \in \Omega_{d}} u(i, j, t)\right]^{2}
$$

where $\Omega_{d}$ and $N_{d}$ are the set and the number of the $2 \mathrm{D}$ points in the diffuse region image, $u(i, j, t)$ is defined as

$$
u(i, j, t)=\frac{I(i, j) r(i, j, t)^{2}}{\cos \theta_{i}(i, j, t)}, \quad(i, j) \in \Omega_{d}
$$

${ }^{1}$ Because of the off-specular peak, the point $P$ will not be the specular peak. However, it will be sufficiently close for practical purposes except at the grazing angles. where $I(i, j)$ is the pixel value observed at 2D point $(i, j)$ in the original image, $r(i, j, t)$ is the distance between the light source and the surface point of 2D point $(i, j)$ under the light source distance $t$, and $\theta_{i}(i, j, t)$ is the incident angle at this surface point.

Since (8) is difficult to solve analytically, we search the optimal source distance $t^{*}$ in the finite, discrete solution space $\Omega_{t}=\left\{t_{\min }, t_{\min }+\Delta t, \ldots, t_{\max }-\Delta t, t_{\max }\right\}$, where $t_{\text {min }}, t_{\max }$ and $\Delta t$ are the user-defined lower bound, upper bound and steplength with respect to $t$, respectively.

Second, we estimate the diffuse reflection parameters $K_{d}$ using $t^{*}$ by minimizing the squared error as

$$
K_{d}^{*}=\underset{K_{d}}{\operatorname{argmin}} \sum_{(i, j) \in \Omega_{d}}\left[I(i, j)-\frac{K_{d} \cos \theta_{i}\left(i, j, t^{*}\right)}{r\left(i, j, t^{*}\right)^{2}}\right]^{2}
$$

The solution $K_{d}^{*}$ can be expressed as

$$
K_{d}^{*}=\sum_{(i, j) \in \Omega_{d}} I(i, j) / \sum_{(i, j) \in \Omega_{d}} \frac{\cos \theta_{i}\left(i, j, t^{*}\right)}{r\left(i, j, t^{*}\right)^{2}}
$$

\subsection{Separating-and-Fitting Relaxation}

The specular component of the input image can be obtained by subtracting the aforementioned diffuse estimate from the input image. The pixel value at point $(i, j)$ in the specular image can be computed as

$$
I_{s}^{*}(i, j)=I(i, j)-\frac{K_{d}^{*} \cos \theta_{i}\left(i, j, t^{*}\right)}{r\left(i, j, t^{*}\right)^{2}}, \quad(i, j) \in \Omega
$$

To accomplish fitting of the specular reflection model to the residual image $I_{s}^{*}$, we need to solve a nonlinear optimization problem as

$$
\begin{array}{r}
\underset{K_{s}, \sigma}{\operatorname{argmin}} \sum_{(i, j) \in \Omega}\left(I_{s}^{*}(i, j)-\frac{K_{s}}{r\left(i, j, t^{*}\right)^{2} \cos \theta_{r}(i, j)}\right. \\
\left.\times \exp \left[-\frac{\alpha\left(i, j, t^{*}\right)^{2}}{2 \sigma^{2}}\right]\right)^{2}
\end{array}
$$

To obtain desirable solutions, we use a two-step algorithm. The details are as follows.

1) Initial estimation of $\left(K_{s}, \sigma\right)$ : we rewrite the specular reflection model as

$$
I_{s}(i, j, t)=\frac{K_{s}}{r(i, j, t)^{2} \cos \theta_{r}(i, j)} \exp \left[-\frac{\alpha(i, j, t)^{2}}{2 \sigma^{2}}\right]
$$

In order to solve (13), the following equation can be derived by the logarithmic transformation of (14):

$$
y(i, j)=-\frac{1}{\sigma^{2}} x(i, j)+\ln K_{s}, \quad(i, j) \in \Omega
$$

where

$$
\begin{aligned}
& x(i, j)=\frac{\alpha\left(i, j, t^{*}\right)^{2}}{2} \\
& y(i, j)=\ln \left[I_{s}^{*}(i, j) r\left(i, j, t^{*}\right)^{2} \cos \theta_{r}(i, j)\right]
\end{aligned}
$$


by which we can plot a set of data pairs $(x(i, j), y(i, j))$, $(i, j) \in \Omega$. By line fitting on these 2D points based on the least-squares method, and then by comparing the coefficients of the regression line with (15), we obtain the initial estimates $K_{s}^{*}$ and $\sigma^{*}$ of the specular parameters as

$$
K_{s}^{*}=\exp (b), \quad \sigma^{*}=\sqrt{-\frac{1}{a}}
$$

where $a<0$ and $b$ are, respectively, the slope and Yintercept of the least-squares regression line.

2) Refinement of $\left(K_{s}, \sigma\right)$ : The estimated specular reflection parameters in the first step are based on the logarithm fitting; thus, the synthesized image based on the initial estimates $\left(K_{s}^{*}, \sigma^{*}\right)$ is still quite different from the input image. In this step, we search for the solution of (13) locally around the initial guesses $\left(K_{s}^{*}, \sigma^{*}\right)$ through a two-fold iteration algorithm as follows.

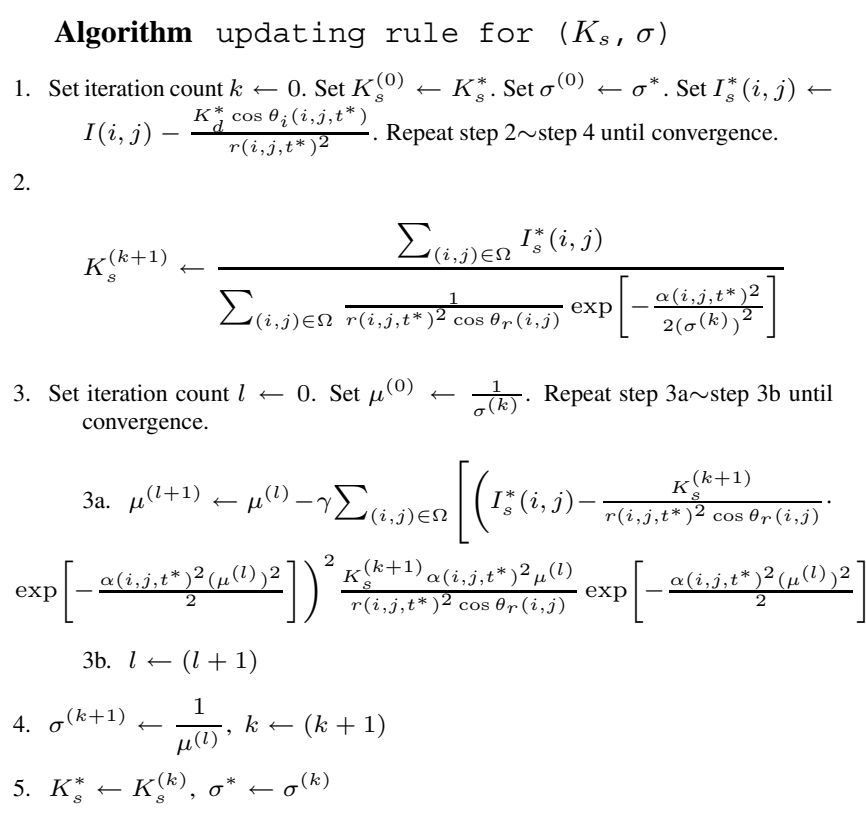

Note that the step size $\gamma$ needs to be set to a sufficiently small value (we used $\gamma=1.0 \times 10^{-7}$ ). Using the above algorithm, we can obtain the estimates $\left(K_{s}^{*}, \sigma^{*}\right)$ of the specular reflection parameters. However, $\left(K_{d}^{*}, K_{s}^{*}, \sigma^{*}, t^{*}\right)$ are still inaccurate, since they are computed based only on the rough diffuse regions. Therefore, we have to update the diffuse reflection component, as described in the following equations:

$$
I_{d}^{*}(i, j)=I(i, j)-\frac{K_{s}^{*}}{r\left(i, j, t^{*}\right)^{2} \cos \theta_{r}(i, j)} \exp \left[-\frac{\alpha\left(i, j, t^{*}\right)^{2}}{2\left(\sigma^{*}\right)^{2}}\right]
$$

After that, we re-estimate the light source distance and diffuse reflection parameters as:

$$
\underset{K_{d}, t \in \Omega_{t}}{\operatorname{argmin}} \sum_{(i, j) \in \Omega}\left[I_{d}^{*}(i, j)-\frac{K_{d} \cos \theta_{i}(i, j, t)}{r(i, j, t)^{2}}\right]^{2}
$$

We can solve (19) through an iteration algorithm as follows.

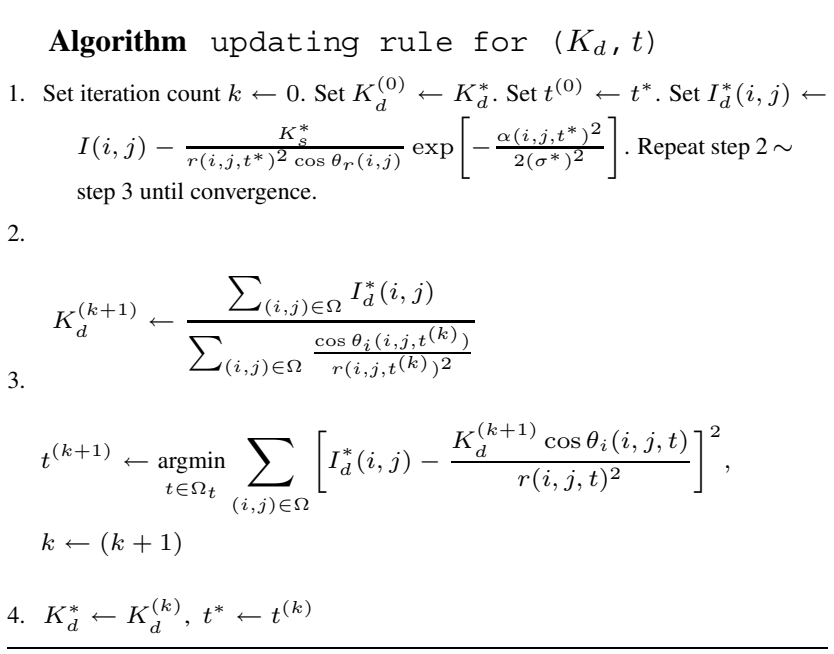

Finally, the above two algorithms are repeated alternately until the reflectance parameters and light source position become no longer changed in values or until they reach the maximum number of iterations. Using $t^{*}$, we can obtain the light source position $\boldsymbol{L}^{*}$ by using (6).

\section{Inverse Rendering from a Single Specular Image}

A major limitation of the algorithm of the last section is its homogeneous Lambertian approximation of the diffuse reflection. In order to avoid this restriction, our second method takes as input a single specular image instead of a single color one, except for a 3D geometric model of an object. Also, the first technique assumed that the specular peak could be identified, although invisible specular peaks or saturated pixels are not uncommon. We also remove this constraint in the second method.

The important thing to note here is that higher $t$, as well as higher $\sigma$, generate a more blurred specular reflection ( $t$ is the light source distance and $\sigma$ is the surface roughness) and hence, $(t, \sigma)$ seems not to be determined uniquely from only a single specular image, which makes this inverse problem ill-conditioned or ill-posed. However, in practice, $(t, \sigma)$ can be determined uniquely from a given specular image, however, due to space limitations the proof is omitted.

In this paper, we describe how polarization filters are used for separating the diffuse and specular reflection components. One is placed in front of a point light source in order to polarize the light source linearly, and the other is placed in front of a camera to capture images through the linear polarization filter. For separating the diffuse and specular reflection components, images of a target object are taken at every certain degrees of filter rotation. Then, the maximum intensity $I_{\max }$ and the minimum intensity 
$I_{\min }$ are determined for every image pixel. If $I_{\max }-I_{\min }$ for a certain pixel is less than the threshold, we consider the pixel to contain only the diffuse component. We determine that the pixel contains the specular component and the specular component intensity is obtained from $I_{\max }-I_{\min }$. $I_{m i n}$ is used for determining the diffuse component intensity. (Technical details are omitted in order to keep this paper within a reasonable length. Interested readers can refer to [13].)

In this method, we assume, (1) camera parameter is known, (2) interreflection and shadows are avoided, (3) the specular reflectance property can be uniformly modeled according to the T-S model (e.g., dielectric material).

\subsection{Log-Linearized T-S Model-Based Recovery}

In this section, we explain how to estimate the specular reflectance parameters as well as the source position from a single specular image $I_{s}=I_{\max }-I_{\min }$. As stated in Section 3.1, a three dimensional source position is represented here by a twofold set $\langle p, t\rangle$, where $p=\left(i_{p}, j_{p}\right)$ is the image pixel location of the specular peak satisfying $\alpha=0$, and $t$ is the source distance. The source position is discretized in the solution space $\langle p, t\rangle$ with a Cartesian product $\Omega_{p} \times \Omega_{t}$, where $\Omega_{p}$ and $\Omega_{t}$ are the user-defined finite, discrete search spaces with regard to $p$ and $t$, respectively. At this stage, we search for the optimal values of $\langle p, t\rangle$ by a discrete optimization technique, whereas the method of Section 3 does only the source distance $t$ for a fixed $p$

Assuming that $\langle p, t\rangle$ is unknown, the linearized reflection model can be obtained based on the logarithmic transformation described in Section 3.3 as:

$$
Y(i, j, p, t)=-\frac{1}{\sigma^{2}} X(i, j, p, t)+\ln K_{s}, \quad(i, j) \in \Omega
$$

where

$$
\begin{aligned}
X(i, j, p, t) & =\frac{\alpha(i, j, p, t)^{2}}{2} \\
Y(i, j, p, t) & =\ln \left[I_{s}(i, j) r(i, j, p, t)^{2} \cos \theta_{r}(i, j)\right]
\end{aligned}
$$

Based on (20), our basic idea is to find $\left\langle p^{+}, t^{+}\right\rangle$that yields the strongest negative linear relationship between $X(i, j, p, t)$ and $Y(i, j, p, t)$ by minimizing a correlation coefficient as

$$
\begin{aligned}
& \underset{(p, t) \in \Omega_{p} \times \Omega_{t}}{\operatorname{argmin}}[ \\
& \left.\frac{\sum_{(i, j) \in \Omega}(X(i, j, p, t)-\bar{X}(p, t))(Y(i, j, p, t)-\bar{Y}(p, t))}{\sqrt{\sum_{(i, j) \in \Omega}(X(i, j, p, t)-\bar{X}(p, t))^{2}} \sqrt{\sum_{(i, j) \in \Omega}(Y(i, j, p, t)-\bar{Y}(p, t))^{2}}}\right]
\end{aligned}
$$

where $\bar{X}(p, t)$ and $\bar{Y}(p, t)$ are sample means:

$\bar{X}(p, t)=\frac{1}{N} \sum_{(i, j) \in \Omega} X(i, j, p, t), \quad \bar{Y}(p, t)=\frac{1}{N} \sum_{(i, j) \in \Omega} Y(i, j, p, t)$

where $N$ is the number of the $2 \mathrm{D}$ points belonging to $\Omega$.

To solve (23), we apply a similar discretization approach to the one mentioned in 3.2. Once the optimal solution $\left(p^{+}, t^{+}\right)$is obtained, the specular reflection parameter $\left(K_{s}^{+}, \sigma^{+}\right)$can be calculated from (17).

\subsection{Three-Step Numerical Optimization}

Since the specular reflection parameter estimation in the last section is based on the logarithmic fitting, the image resulting using the estimates $\left(K_{s}^{+}, \sigma^{+}, p^{+}, t^{+}\right)$is still too deviated. In this stage, we formulate a nonlinear optimization problem with respect to $K_{s}, \sigma, p$ and $t$ as:

$$
\begin{array}{r}
\underset{K_{s}, \sigma,(p, t) \in \Omega_{p} \times \Omega_{t}}{\operatorname{argmin}} \sum_{(i, j) \in \Omega}\left(I_{s}(i, j)-\frac{K_{s}}{r(i, j, p, t)^{2} \cos \theta_{r}(i, j)}\right. \\
\left.\times \exp \left[-\frac{\alpha(i, j, p, t)^{2}}{2 \sigma^{2}}\right]\right)^{2}
\end{array}
$$

Note that $r$ and $\alpha$ are also redefined as functions of $(i, j, p, t)$. To solve (24), we apply a 3-step iterative algorithm using $\left(K_{s}^{+}, \sigma^{+}, p^{+}, t^{+}\right)$as the initial guess. The procedure is described in the following.

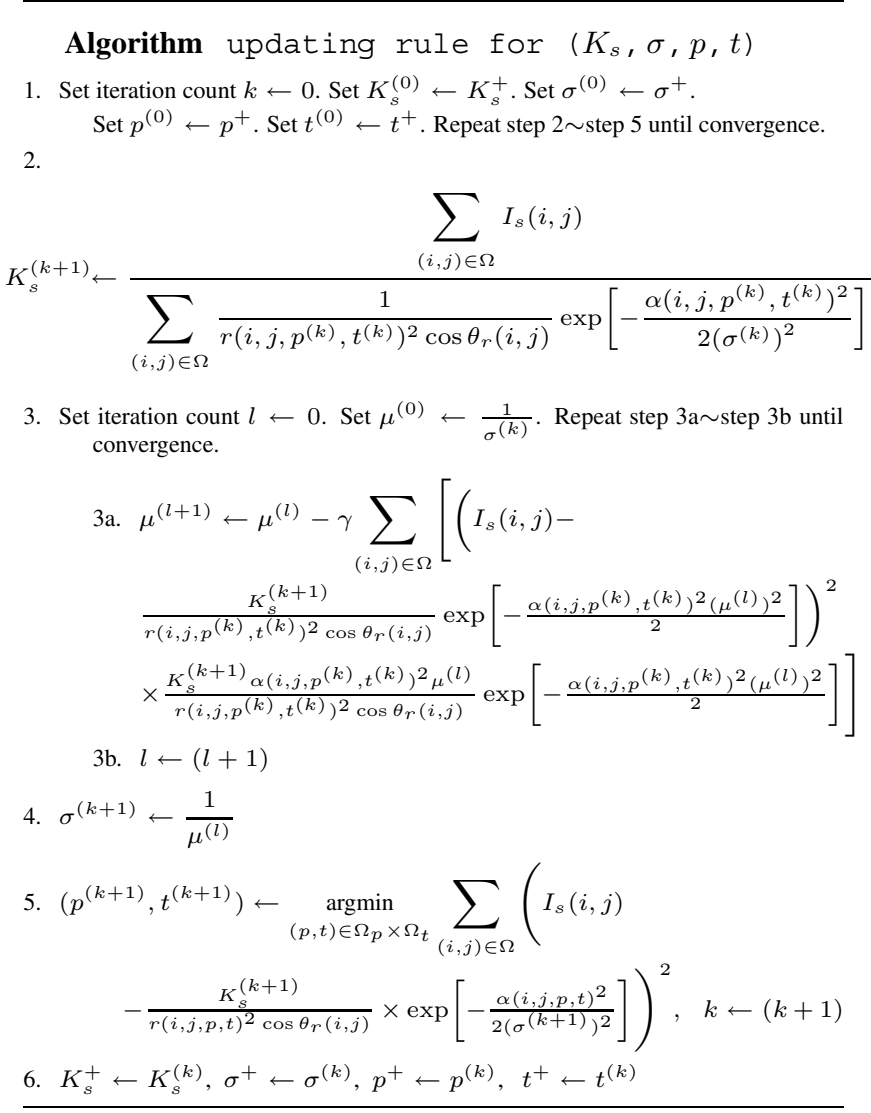




\subsection{Diffuse Parameter Estimation}

Unlike the method of Section 3, the method of this section does not directly estimate the diffuse reflection parameters. However, to synthesize new images under novel illumination conditions, we need to determine those values according to some appropriate diffuse reflection model. In the case of textured surfaces with specular reflection, we determine the Lambertian parameters for each pixel, i.e., texture, as

$$
K_{d}^{+}(i, j)=\frac{r\left(i, j, p^{+}, t^{+}\right)^{2} I_{d}(i, j)}{\cos \theta_{i}\left(i, j, p^{+}, t^{+}\right)}, \quad(i, j) \in \Omega
$$

where $I_{d}$ is the separated diffuse image and $\theta_{i}$ is the incident angle. $p^{+}$and $t^{+}$are, respectively, the estimated specular peak and light source distance in the last subsection. Note that shadowing is not considered in our method.

\subsection{Multiple Light Sources}

Until now, the illumination was assumeded to consist of a single point light source. Taking as input a single image taken under multiple light sources, the solution space becomes too large to be searched robustly within our framework. However, if we can obtain the specular image generated only under a point light source of the set, we can estimate the specular reflection parameters, as well as the three dimensional position of this light source by using the method of this section. In the following, we explain briefly how to separate this specular image.

We use two linear polarization filters: one is placed in front of the camera, and the other is placed in front of the target light source. Now, we rotate the polarization filter of this light source. Note that we must fix the rotation angle of the polarization filter of the camera. Then it is clear that we can obtain the specular image only under the target light source by calculating $I_{\max }-I_{\min }$, assuming that incident light from any other source does not pass through the plarization filter of this light source and that the interference between rays becomes negligible.

\section{Experiments}

In this section, we present results of several experiments using real images. In these experiments, color images are captured using a color CCD video camera and 3D geometric models are obtained using a light-stripe range finder with a liquid crystal shutter. A halogen lamp is used as a light source. The lamp is small enough for us to assume that it is a point light source.

\subsection{Recovery from Real Color Images}

\section{1) Curved surface:}

Fig.2(a) shows the input real image of $\boldsymbol{L}=$ $(-40.88,-49.36,80.48) \quad(\mathrm{cm})$. We obtain $\boldsymbol{L}=$ $(-40.04,-48.83,79.77)(\mathrm{cm}), \tilde{K}_{s}=(0.123,0.0258,0.249)$, $\sigma=0.0702$, and $\tilde{K}_{d}=(0.494,0.840,0.576)$. Fig.2(b) shows the synthetic image obtained using these light source and reflectance parameters.

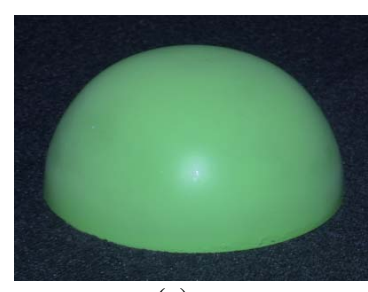

(a)

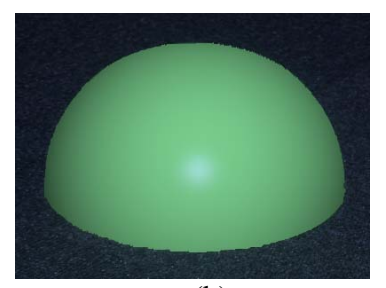

(b)
Figure 2. Input and synthesized image: (a)input color image; (b)synthesized image.

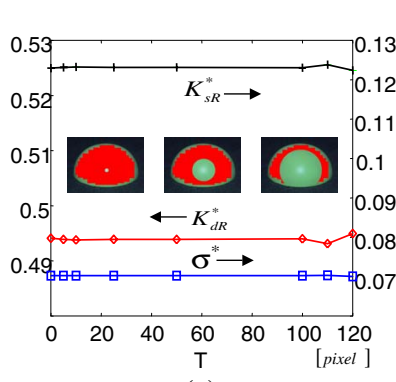

(a)

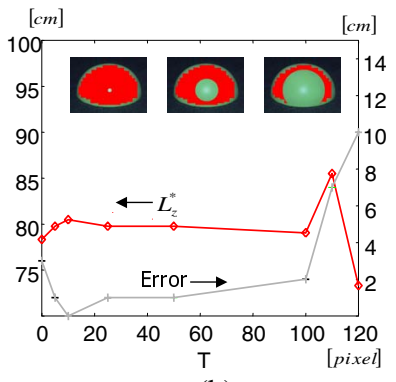

(b)
Figure 3. Stability and error analysis: (a)reflectance parameter estimation; (b)light source position estimation.

We illustrate the variation of the estimates of reflection parameters (Fig.3(a)) and light source position (Fig.3(b)) with respect to variations of the diffuse region. The region is manually extracted at the initial stage as explained in Section 3.2. The horizontal axes in Fig.3(a) and (b) represent the selected threshold value $t h$ for the diffuse region extraction. The left and right vertical axes in Fig.3(a) represent $K_{d, R}$ and $\left(K_{s, R}, \sigma\right)$, respectively. The left and right vertical axes in Fig.3(b) represent the z-coordinate and error of the estimates of $\boldsymbol{L}$, respectively. As can be seen in Fig.3(a) and (b), the estimates of reflection parameters and the light source position are almost constant with respect to variations of the diffuse region, meaning that the manual initial estimation of diffuse regions does not affect the final estimate.

\section{2) Planar surface:}

Fig.4(a) shows an image of a real bookend. By applying our method described in Section 3 to this color image, we obtain the synthetic image (Fig.4(a)). Fig.5(a)-(b) show the synthesized images under shorter and longer distances 


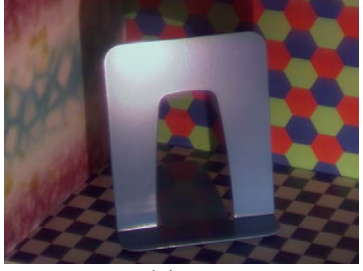

(a)

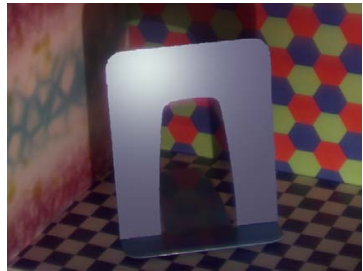

(b)
Figure 4. Input and synthesized images: (a)input color image; (b)synthesized image.

of light source position than that of the above, respectively. Fig.5(c) shows the synthesized image under a different light source direction. Fig.5(d) shows the synthesized image under a different distance and a different color (we used an illuminant color estimation method [14]) of the light source.

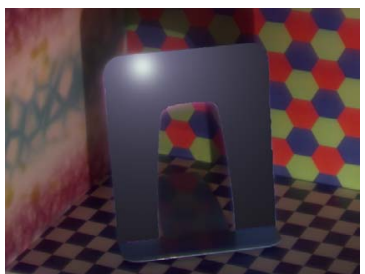

(a)

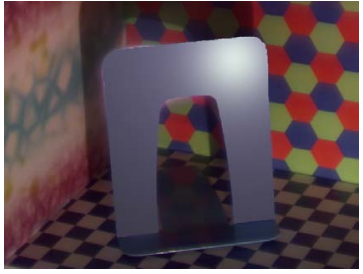

(c)

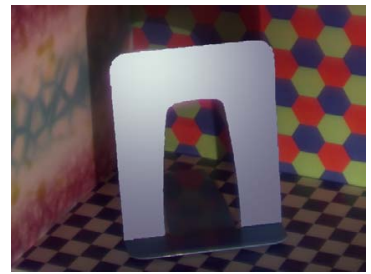

(b)

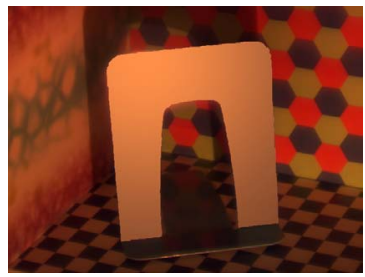

(d)
Figure 5. Synthesized images under novel illumination conditions: (a)shorter source-surface distance; (b)longer source-surface distance; (c)different source direction; (d)longer source-surface distance and different source color.

\subsection{Recovery from Real Specular Images}

In this section, we describe how we use as input the separated specular image using polarization filters.

1) Textured surface (specular peak is invisible):

Fig.6(a) shows a textured object: a poly-coated board. We apply our method of Section 4 to this object under the assumption of a perfectly uniform specular reflectance. Fig.6(b) and Fig.6(c) show, respectively, the diffuse and specular reflection image separated using polarization. Fig.7(a) shows the recovered image. Fig.7(b) shows the synthesized image under a different light position. Note that the diffuse reflection components are recovered by using (25). Fig. 8 shows a plot with the light source distance on the horizontal axis and the correlation coefficient (23) on the vertical axis, for a $\left(i_{p}, j_{p}\right)$ value, in the initial estimation stage. Each graph in this figure shows $2 \mathrm{D}$ plot with $X$-axis and $Y$-axis defined in (21) and (22), respectively.

In the second-stage final estimation, we obtain $\boldsymbol{L}=$ $(3.31,-111.51,106.0)(\mathrm{cm}), \tilde{K}_{s}=(0.246,0.297,0.216), \sigma=$ 0.115 , where the ground truth for the source position is $\boldsymbol{L}=(0.0,-108.9,99.0)(\mathrm{cm})$. The reasons why the estimated source position is slightly erroneous are considered to be as follows: 1) the target object has a transparent coat and thus reflection also occurs under the transparent coat, 2) the specular peak is invisible in the input image and the image pixel location of the specular peak is erroneous.



(a)

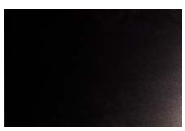

(b)

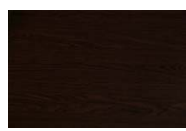

(c)
Figure 6. Input specular image: (a)original image; (b)specular image; (c)diffuse image.

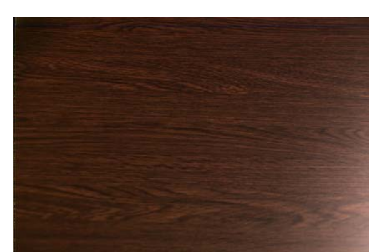

(a)

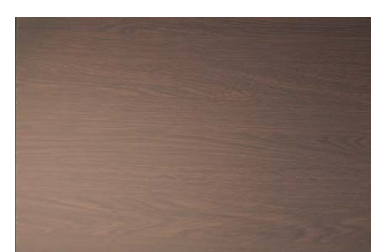

(b)
Figure 7. Synthesized image: (a)original light position; (b)new light position.

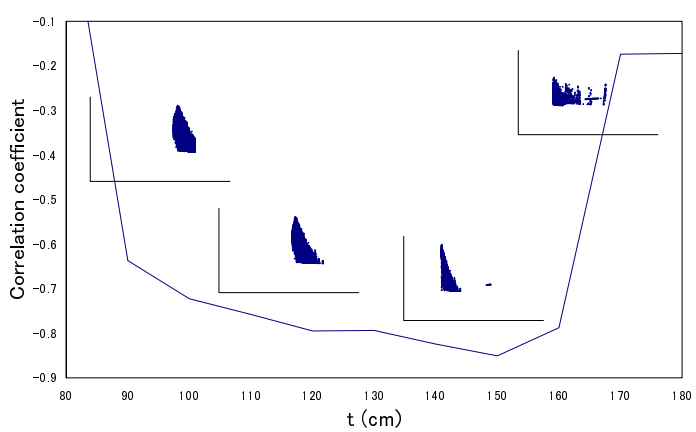

Figure 8. Relationship between light source distance and correlation coefficient. 


\section{2) Multiple light sources:}

Fig.9(a) shows the original image of a real object under multiple light sources. We separate the specular image under a target light source (specular highlight in the upper part of the original image) of illumination using polarization filters, as shown in Fig.9(b). Fig.9(c) shows the residual image (original image - specular image). We obtain $\boldsymbol{L}=(4.37,-74.1,17.3)(\mathrm{cm}), \tilde{K}_{s}=(0.137,0.163,0.169)$, $\sigma=0.0731$. Fig.9(d) shows the synthesized image.

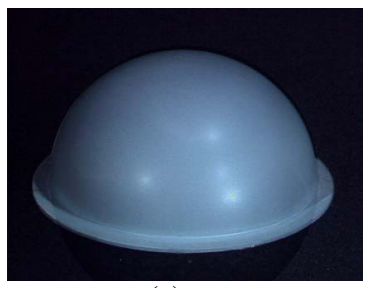

(a)

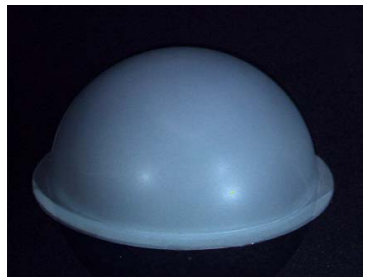

(c)

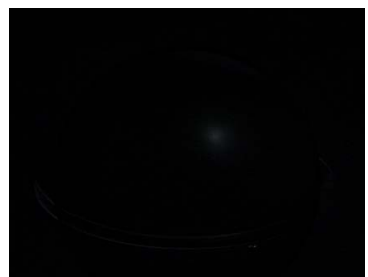

(b)

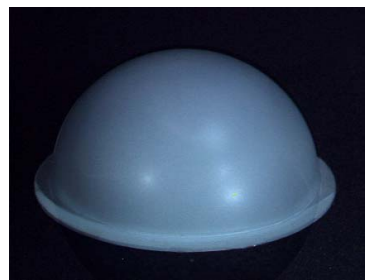

(d)
Figure 9. Real and synthesized images: (a)original image; (b)specular image under target light source; (c)residual image; (d)synthesized image.

\section{Conclusions}

In this paper, we have presented two new methods for recovering the surface reflectance properties of an object and the light source position from a single image without the distant illumination assumption; hence, those methods allow the image synthesis of the target object under arbitrary light source positions. In particular, the second method can also deal with textured objects with specular reflection. The first method assumes that the object has a homogeneous Lambertian diffuse reflection property. The second method does not Make these assumptions, and thus can handle diffuse textured objects; however, it is less robust than the first method because no information about diffuse reflection is available. We intend to extend the current method to the cases of erroneous geometric models in future work.

\section{References}

[1] K. Ikeuchi and K. Sato, "Determining reflectance properties of an object using range and brightness images," IEEE
Trans. Pattern Analysis and Machine Intelligence, Vol.13, No.11, pp.1139-1153, 1991.

[2] S.R. Marschner and D.P. Greenberg, "Inverse lighting for photography," Proc. the Fifth Color Imaging Conference. Society for Imaging Science and Technology, pp.262-265, 1997.

[3] R. Ramamoorthi and P. Hanrahan, "A signal processing framework for inverse rendering," Proc. SIGGRAPH'01, pp.117-128, 2001.

[4] K. Nishino, "Photometric object modeling -rendering from a dense/sparse set of images-," Ph.D thesis, The University of Tokyo, 2002.

[5] S. Tominaga and N. Tanaka, "Estimating reflection parameters from a single color image," IEEE Computer Graphics and Applications, Vol.20, No.5, pp.58-66, 2000.

[6] I. Sato, Y. Sato, and K. Ikeuchi, "Illumination distribution from brightness in shadows: adaptive estimation of illumination distribution with unknown reflectance properties in shadow regions," Proc. ICCV'99, pp.875-882, 1999.

[7] S. Boivin and A. Gagalowicz, "Image-based Rendering of diffuse, specular and glossy surfaces from a single image," Proc. SIGGRAPH'01, pp.197-216, 2001.

[8] S. Shafer, "Using color to separate reflection components," COLOR Research and Application, Vol. 10, pp. 210-218, 1985.

[9] L.B. Wolff and T.E. Boult, "Constraining object features using a polarization reflectance model," IEEE Trans. Pattern Analysis and Machine Intelligence, Vol.13, No.6, pp.1671891991.

[10] Y. Sato, M. Wheeler, and K. Ikeuchi, "Object shape and reflectance modeling from observation," Proc. SIGGRAPH'97, pp.379-387, 1997.

[11] T.E. Boult and L.B. Wolff, "Phisically based edge labeling," Proc. Computer Vision and Pattern Recogition, pp.656-663, 1991.

[12] K.E. Torrance and E.M. Sparrow, "Theory of off-specular reflection from roughened surfaces," Journal of the Optical Society of America, Vol.57, pp.1105-1114, 1967.

[13] L.B. Wolff and T.E. Boult, "Costraining object features using a polarization reflectance model," IEEE Trans. Pattern Analysis and Machine Intelligence, Vol.13, No.6, pp.6356571991.

[14] G.D. Finlayson and G. Schaefer, "Solving for color constancy using constrained dichromatic reflection model," Int. J. of Computer Vision, Vol.42, No.3, pp.127-144, 2001. 